\title{
AC Magnetic Susceptibility Technique for the Characterization of High Temperature Superconductors
}

\author{
M. I. Youssif, A. A. Bahgat" and I. A. Ali \\ Physics Department, Faculty of Science- Damietta, Mansoura University, New \\ Damietta, Egypt. \\ *Physics Department, Faculty of Science, Al-Azhar University, Nasr City \\ 11884, Cairo, Egypt.
}

The absolute magnetic susceptibility as a function of temperature with different amplitude and frequency of the applied field is used to characterize the magnetic properties of high $T_{c}$-superconductors. In the present work our design of a laboratory-built ac susceptometer based on the mutual inductance method using local facilities is demonstrated and tested. The setup have moment precision of better than $7 \times 10^{-10} \mathrm{~A} \cdot \mathrm{m}^{2}$. The real, or in-phase, component and the imaginary, or out of-phase, component of the complex susceptibility were measured independently applying a computer program designed specially for this purpose. $\mathrm{YBa}_{2} \mathrm{Cu}_{3} \mathrm{O}_{7-x}$ high temperature ceramic superconductor was used to test and calibrate the system. The formulas for susceptibilities corresponding to the models of linear diffusion and critical state are used in the recognition of the mechanism controlling the flux dynamics and in the determination of the superconducting properties for $\mathrm{YBa}_{2} \mathrm{Cu}_{3} \mathrm{O}_{7-x}$ and $\mathrm{EuBa} \mathrm{Cu}_{3} \mathrm{O}_{7-x}$. Superconducting properties such as, critical temperature, $T_{c-\text {-onset }}$ critical current density, $J_{c}$, and electrical resistivity were calculated from the experimental data. 


\section{Introduction}

The importance of magnetic property measurements to the characterization of high $T_{c}$ superconductors has generated increased interest in the use of this science. Magnetic properties can be measured in either ac or dc magnetic fields. The magnetic measurements differ from the resistivity measurements in the following ways: (1) specimens do not require electrical contacts, but can be small or even in the powder form; (2) A magnetic signal is given at temperatures below $\mathrm{T}_{\mathrm{c}}$, when resistivity $\rho \rightarrow 0$, so the magnetic signal can be used to characterize the material at low temperatures; (3) The superconducting volume fraction can be estimated; (4) The signal is given even if the superconducting path is not continuous; (5) The critical current density $\mathrm{J}_{\mathrm{c}}$ 's can be measured independently of contacts and of intergranular weak links.

The dc magnetometer and the ac susceptometer are two entirely different tools that provide different ways of examining magnetic properties. Both these techniques relay on sensing coils used to measure the variation in the magnetic flux due to magnetized sample. The difference between these two techniques resides in how the flux variation is achieved. In a dc magnetization measurement[1], a sample is subjected to and magnetized by a static dc field usually arising from an iron core, or superconducting magnet. The sample is then moved relative to a detection coil that is used to measure variations in flux due to the magnetized sample. The output signal from the detection coil can be measured and related to the materials magnetic properties. On the other hand, in ac measurements ${ }^{(2,3)}$, the sample is centered within a coil while driven with an external ac field. This produces a time-varying magnetization that a second detection coil senses. Usually a lock-in amplifier is used to analyze this signal is related to the materials ac magnetic properties. Alternatively, superconducting quantum interface device, SQUID magnetometers, and vibrating sample magnetometers, VSM, are somewhat similar in purpose; both measure the magnetic moment of the sample, and are being used in many laboratories[4].

An ac susceptometer is however a unique tool among these instruments. Where it is capable of measuring susceptibility under very small ac magnetic fields, with or without a dc bias field. It can also measure the magnetic susceptibility as a function of frequency and temperature, while it is capable to separate the real component $\chi^{\prime}$, and the imaginary component $\chi$ " of the complex susceptibility. AC susceptibility measurements have been employed to qualify the suitability of materials as superconductors[2].

Magnetic properties of materials have been extensively studied using ac susceptometers with varying configurations. A simple registration of the ac 
susceptibility is enough to characterize the type I superconductor[5]. While for the development of the type II superconductors more advanced experimental methods are needed to study the volume pinning force and the surface barriers[6]. Filamentary superconductivity[7], effects of granularity[8] and the importance of correlating the ac susceptibility data with the structure were pointed out as an ac susceptibility active area of research. Moreover, the ac losses are probably one of the most important topics related to the ac susceptibility[9]. Recently the wide-band ac susceptibility technique was developed $^{(10)}$ and could serve as an integrating concept for all these methods.

In the present paper the design of a homemade ac susceptometer is given. The design depends on local facilities and is based on the mutual inductance method. We have used a system of two identical sensing coils (secondary) oppositely wound, connected in series to detect the variation in flux due to the sample positioned in the center of one of them. The lock-in technique was used to register the sample's induced magnetic response sensed by the pick-up coil. Measuring the temperature dependence of the absolute magnetic susceptibility is employed to characterize the magnetic properties of $\mathrm{EuBa}_{2} \mathrm{Cu}_{3} \mathrm{O}_{7-\delta}$ of the high $\mathrm{T}_{\mathrm{c}}$ superconductors. Results of the basic physical properties of our specimens from the ac data (resistivity, critical temperatures, and critical current density) are then discussed.

\section{Theoretical background}

\section{Definitions and performance comparison}

For the measurement techniques described below, the starting point is the magnetic moment " $\mathrm{m}$ ". Magnetic moment is usually associated with the orbital motion and/ or the spin motion of the charged particles in the material, and is a measure of the magnetic field generated by the sample itself. While magnetization originates from permanently magnetized materials or by magnetizing samples through the application of an applied magnetic field. When comparing different materials, or samples of different sizes, the macroscopic quantity of interest is the magnetization or net magnetic moment per unit volume, or per unit mass. The volume magnetization $M$ is defined as:

$$
\mathrm{M}=\mathrm{m} / \mathrm{V}
$$

However, the magnetic susceptibility, $\chi$, of an isotropic substance is defined as: "the intensity of magnetization acquired by an infinitely thin bar placed lengthwise in a uniform field of unit magnetic force". The specification of an infinitely thin bar eliminated the need to consider demagnetizing fields[11]. 
The condition of a field of unit magnetic force defined susceptibility as the ratio of magnetization $M$ to magnetic field strength $H$ :

$$
\chi=\mathrm{M} / \mathrm{H}
$$

That is, the magnetic susceptibility $\chi$ is a measure of how "magnetic" a material is and represents the response of the material to an applied field $\mathrm{H}$. Although the volume susceptibility is a dimensionless parameter, the actual numerical value will depend on the system of units being used.

In a dc magnetization measurement a value for the magnetic moment $\mathrm{m}$ is measured for some applied dc field, $\mathrm{H}_{\mathrm{dc}}$ [1]. Where if the sample being measured does not have a permanent magnetic moment, an applied field is required to magnetize the sample. Magnetization and the dc susceptibility both are derived quantities. Usually the moment is measured, as a function of field, and the materials magnetization curve (that is, $m$ or $M$ versus $H_{d c}$ ) is determined. A detection coil is used to detect the change in magnetic flux due to the presence of the magnetic moment. Since the applied magnetic field is constant, there will be no signal associated with $\mathrm{H}_{\mathrm{dc}}$ (Faraday's law). The sample flux coupled to the detection coil is made to vary by moving the sample. The dc or static susceptibility is determined from equation (2) by dividing the magnetization by the applied field:

$$
\chi_{\mathrm{dc}}=\mathrm{M} / \mathrm{H}_{\mathrm{dc}}
$$

As in the dc magnetometer, an ac susceptometer uses a detection coil to detect changes in the magnetic flux due to the sample. The main difference lies in how the flux variation is achieved.

On the other hand, in an ac susceptometer the sample is generally centered within a detection coil and driven with an applied ac magnetic field $\mathrm{H}_{\mathrm{ac}}[3,11]$. The magnetic moment of the sample will follow the applied field cycle. The detection circuitry is generally balanced with a second identical, but oppositely wound, empty coil to null out the flux changes related to $\mathrm{H}_{\mathrm{ac}}$. As a result, any experimentally detected change in flux is due only to the changing moment of the sample (dm) as it responds to the ac field (no sample movement is required to produce an output signal) and not to the moment itself as in dc technique. The ac susceptibility obtained is similar to eqns. (1) and (2):

$$
\chi_{\mathrm{ac}}=\mathrm{dm} / \mathrm{VH}_{\mathrm{ac}} \rightarrow \mathrm{dM} / \mathrm{dH}
$$

Thus, the ac susceptibility is actually the slope $(\mathrm{dM} / \mathrm{dH})$ of the magnetization curve ( $\mathrm{M}$ versus $\mathrm{H}$ curve). The ac technique detects changes in the magnetization that lead to $\mathrm{dM} / \mathrm{dH}$ in the limit of small ac fields, and this is 
why sometimes referred to as a differential susceptibility. This is the fundamental difference between the ac and dc measurement techniques.

The real importance of an ac susceptibility measurement lies not in where it overlaps the capabilities of a dc magnetometer, but rather in the additional physics one can learn from the material properties under investigation. To understand this, the distinction between the $\mathrm{dc}$ and the ac measurement must be emphasized.

In the dc measurement, the magnetic moment of the sample does not change with time. Thus, a static magnetic measurement is performed. An ac output signal is detected in a VSM, but this signal arises from the periodic movement of the sample, and is not representative of the ac response of the sample itself. In the ac measurement, the moment of the sample is actually changing in response to an applied ac field, allowing the dynamics of the magnetic system to be studied.

Since the actual response of the sample to an applied ac field is measured, the magnetodynamics can be studied through the complex susceptibility $\left(\chi^{\prime}+i \chi^{\prime \prime}\right)$. The real component $\chi^{\prime}$ represents the component of the susceptibility that is in phase with the applied ac field, while $\chi^{\prime \prime}$ represent the component that is out of phase. The imaginary component $\chi^{\prime \prime}$ is related to the energy losses, or in other words, the energy absorbed by the sample from the ac field.

\section{Theory}

Consider a sample in the form of a slab[2] (long enough to be considered infinite compared with its width) is placed in the magnetic field $\mathrm{B}_{\text {ext }}$ $=\mathrm{B}_{\mathrm{dc}}+\mathrm{B}_{\mathrm{ac}}$ having the static component $\mathrm{B}_{\mathrm{dc}}$ and ac component $\mathrm{B}_{\mathrm{ac}}$ characterized by the frequency $f=\omega / 2 \pi$ and the amplitude $B_{a}$ given by:

$$
\mathrm{B}_{\mathrm{ac}}=\mathrm{B}_{\mathrm{a}} \cos (\omega \mathrm{t})
$$

parallel to the surface of the slab. We suppose that the DC and AC fields are parallel in space, although sometimes the perpendicular arrangement of the fields is used [12].

The magnetic flux crossing the sample area can be expressed in terms of the mean value $\bar{B}$ of the flux density through the sample-cross-section A: 


$$
\Phi_{m}=A \bar{B}=\int_{A} B \cdot d A
$$

In ac experiment $\bar{B}$ is a function of time and controls the voltage in one turn of the pick-up coil:

$$
V_{m}(t)=-\frac{\partial \Phi_{m}(t)}{\partial t}=-A \frac{\partial \bar{B}(t)}{\partial t}
$$

It is important to know that the pick-up coil measures an integrated value of the flux density in the sample. Using eqn. (6) one can define the sample magnetization as:

$$
M(t)=\bar{B}(t)-B_{e x t}(t)=\frac{\Phi_{m}(t)}{A}-B_{e x t}(t)
$$

The complex ac susceptibility components are defined as [7]:

$$
\begin{aligned}
& \chi^{\prime}=\frac{1}{\pi B_{a}} \int_{0}^{2 \pi} M(\omega t) \cos (\omega t) d(\omega t) \\
& \chi^{\prime \prime}=\frac{1}{\pi B_{a}} \int_{0}^{2 \pi} M(\omega t) \sin (\omega t) d(\omega t)
\end{aligned}
$$

The physical meaning of the real component $\chi^{\prime}$ and the imaginary component $\chi^{\prime \prime}$ is the following: The energy converted into heat during one cycle of the AC field [13] is

$$
W_{q}=-2 \pi \chi " \frac{B_{a}^{2}}{2 \mu_{o}}
$$

This expression explains why the lock-in can be used to determine AC losses[14]. Since $\mathrm{W}_{\mathrm{q}}$ is always negative, $\chi^{\prime \prime}$ in a correctly designed experiment must take the positive sign.

The time average of the magnetic energy stored in the volume occupied by the sample [15] is

$$
W_{m}=\chi^{\prime} \frac{B_{a}^{2}}{2 \mu_{o}}
$$


where the normal-state value was taken as the reference level, i.e. $W_{m}\left(T>T_{c}\right)=$ 0 . Diamagnetic behavior leads to reduction of the magnetic field compared with the normal state, reflected in a negative value of $\mathrm{W}_{\mathrm{m}}$. Thus we expect in the case of a superconductor $\chi^{\prime} \leq 0$. In general, $\chi^{\prime}$ reflects the shielding ability, while $\chi^{\prime \prime}$ is measure of the magnetic irreversibility.

\section{Ac - susceptometer design, setup and experimental procedures}

\section{Construction}

A typical ac-susceptometer for measuring the magnetic susceptibility of non-ferromagnetic material is often consisting $[16,17]$ of a primary excitation field coil, a secondary pick-up coil, and a secondary compensation coil (known as three-coil system). Next we will be describing the design made in our laboratory as built for low-frequency ac-susceptibility measurement using local facilities. A delicate coil winding system was built using a special slow synchronous motor coupled to a continuos digital number of turn's indictor. This facilitates the exact evaluation of the even setting number of turns. A coated copper wire of $0.1 \mathrm{~mm}$ diameter was used for the coils. The coils system consists of two identical coaxial mutual-inductance coils. A four coils system was made and is consisting of two identical primary excitation field coils -1200 turns each- and two identical secondary pick-up coils - 600 turns each. The coils were winded around a hollow "I" shaped cylinders, of $1.2 \mathrm{~cm}$ in height. The schematic block diagram of the system is given in Fig. (1) and showing the arrangement of the coils together with specimen and the temperature sensor.

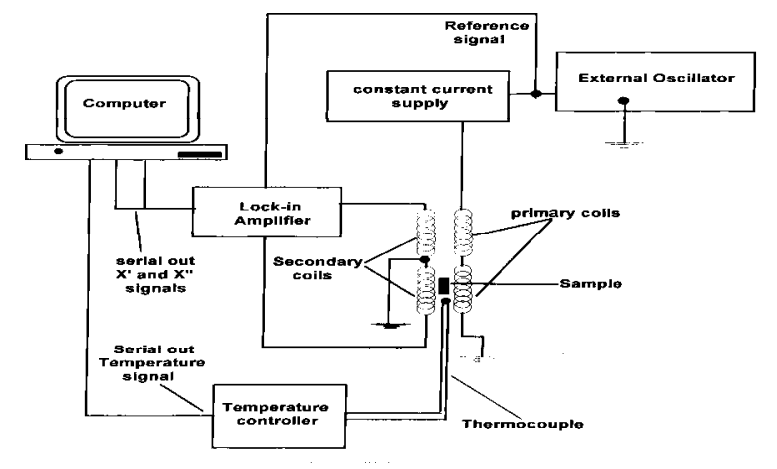

Fig. (1): Block diagram of the experimental setup of the homemade low temperature ac magnetic susceptometer.

The alternating magnetic field is generated by the two identical primary coils connected in series. An ac-constant current source was coupled to an external oscillator was made and used to derive the two identical primary coils connected in series. This permits the measurements of the ac-susceptibility 
over a broad range of discrete field amplitudes, frequencies and temperatures without the need to make any corrections to the field intensity due to changing the ohmic resistance of the coils. The two secondary coils with the midpoint concentric with the primary coils were connected to a common ground. In this design the sample is located at the midpoint of one of the pick-up coils, which was selected so that $\chi$ ' shown negative going below $\mathrm{T}_{\mathrm{c}}$. The balance between the pick-up and reference coils was achieved by winding the mutual inductance of each with respect to the field coil in opposite sense. Here, the measured quantity is ac-permeability: $\mu=\mu^{\prime}+i \mu^{\prime \prime}$ which is numerically related to the acsusceptibility by $\mu=\mu_{0}(1+\chi) . \mu^{\prime}=\mu_{0}\left(1+\chi^{\prime}\right), \mu^{\prime \prime}=\mu_{0} \chi^{\prime \prime}$ where $\mu_{\mathrm{o}}$ is the free space permeability.

While the ac susceptometer relies on inductive coupling between coils, the pick-up coils were winded beneath the field coils with a shield of low temperature silk thread in tape was used to reduce their capacitive coupling [16] and to achieve thermal good stability as well. As the sample is inserted in one of the coils, a change would occur in the self-inductance as well as in the phase, which both are related to the magnetic susceptibility as shown above.

The pick-up and reference coils are connected to the differential input of a computer controlled lock-in amplifier (model SR530, Stanford Research Systems, Inc., Sunnyvale, CA, USA) which is used as an off-balance meter, and is coupled to the computer via a standard serial interface, see Fig. (1).

One of the main critical experimental points of the present setup is the temperature measurement, which should be considered seriously. During the measurement the coil temperature changes with that of the sample by

Fig. (2)

X-ray diffraction patterns of the two prepared ceramic samples, $\mathrm{YBa}_{2} \mathrm{Cu}_{3} \mathrm{O}_{7-\mathrm{x}}$ and $\mathrm{EuBa}_{2} \mathrm{Cu}_{3} \mathrm{O}_{7-\mathrm{x}}$ respectively.

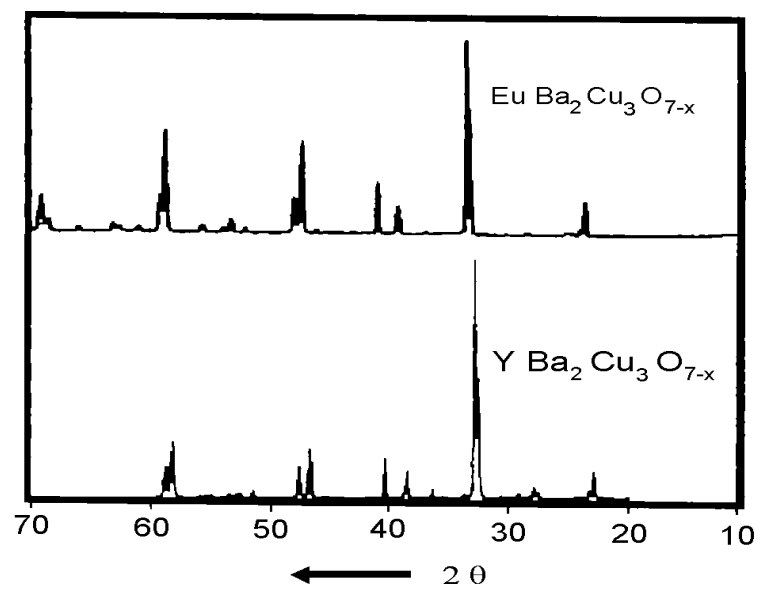

immersing in liquid nitrogen. The normal heat leak in the Dewar is used to 
slowly warm up the sample by the method of heat induction. A copperconstantan thermocouple (type T) was used to measure the sample temperature and is connected to a digital meter, which is coupled to the computer through a serial interface. The position of the thermocouple with respect to the sample should be adjusted carefully to achieve correct temperature measurement. Also it was found a good idea to coat both the sample and the thermocouple with a thin layer of vacuum grease, which act as a good thermal contact agent. The thermocouple thermometer has been calibrated with the standard fixed pointsfreezing point and the boiling point of water, solid $\mathrm{CO}_{2}$, and liquid nitrogen temperatures as well. To truly reflect the actual transition temperature of the specimen under investigation the critical transition temperature, $\mathrm{T}_{c}$, of a standard superconducting sample was used to calibrate the system. Namely, $\mathrm{YBa}_{2} \mathrm{Cu}_{3} \mathrm{O}_{7-\mathrm{x}}$, sample was prepared by the usual ceramic procedure and was tested by our ac- susceptometer, the results are shown in Fig. (3). A transition temperature of $92 \mathrm{~K}$ was detected for this sample, indicating that the sensor was installed at the proper position relative to the sample as shown in Fig. (1).
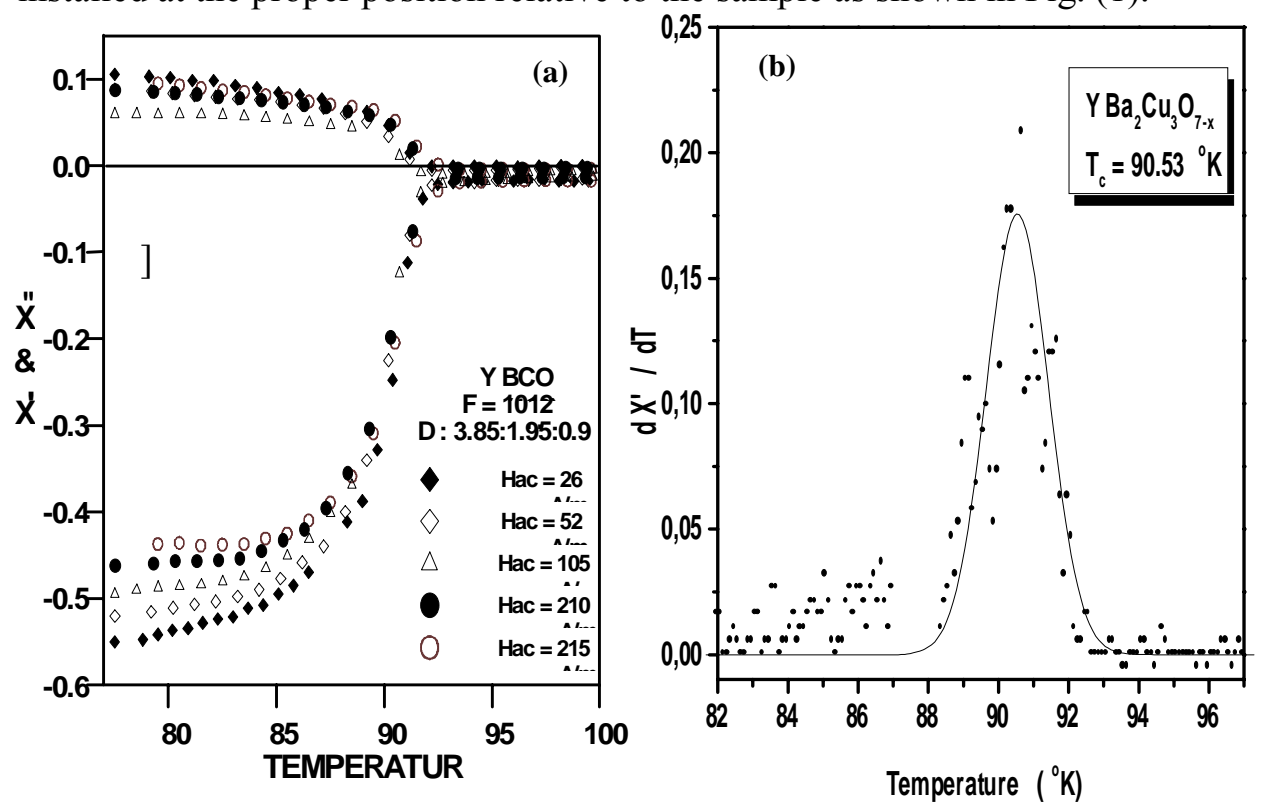

Fig. (3): Ac susceptibility ( $\chi$ ' and $\chi$ ') results for the superconducting $\mathrm{YBa}_{2} \mathrm{Cu}_{3} \mathrm{O}_{7-\mathrm{x}}$ measured at different temperatures and applied magnetic field.

Two samples, $\mathrm{YBa}_{2} \mathrm{Cu}_{3} \mathrm{O}_{7-\mathrm{x}}$ and $\mathrm{EuBa}_{2} \mathrm{Cu}_{3} \mathrm{O}_{7-\mathrm{x}}$ were prepared by the dry method, of heating and sintering in oxygen flow the appropriate constituents. X-ray diffraction was used to identify the final product, which meet the standard patterns, see Fig. (2). The final black pellets were cut and grounded 
into shapes of thin rectangular slabs of dimensions of 3.85:1.95:0.9 $\mathrm{mm}$ to be used during the ac-susceptibility measurements.

\section{Phase Adjustment and Measurements}

The phase angle $\Phi$ of the lock-in amplifier must be adjusted to correctly separate the real and imaginary parts of susceptibility. This adjustment may be done experimentally or theoretically [11]. In the present experiment, the phase difference $\Phi$ between the sample signal and the reference is determined when the sample was in zero loss state - high temperature i.e. above $T_{c}$, where $\chi^{\prime \prime}=0$. Once this phase has been determined, the phasesensitive signal measured at $\Phi$ will be proportional to $\chi^{\prime}$ and the signal measured at $\Phi+90^{\circ}$ will be proportional to $\chi^{\prime \prime}$. Precise phase adjustment is necessary to observe frequency shifts in the susceptibility curves.

On the secondary circuit, the two secondary coils are wound in opposition so that the field induced by the primary produces, after specimen withdrawal, zero potential, $y=0$, measured by the phase-sensitive detector that feeds the lock-in amplifier. When a specimen is centered within one of the sensing coils, the secondary voltage balance is disturbed. The resultant variation in flux produces a measured, emf, voltage $v=-\delta \Phi / \delta \mathrm{t}$, proportional to the susceptibility of the sample. The phase and voltage of the signal received by the lock-in amplifier are fed to the computer, and the signals are recorded as a function of specimen temperature. This susceptibility also depends upon experimental parameters defined by the following relationship:

$$
\boldsymbol{v}_{r m s}=(1 / \alpha) \boldsymbol{V}_{\boldsymbol{s}} \boldsymbol{f} \boldsymbol{H}_{r m s} \chi_{\text {ext }}
$$

where $v_{r m s}$ is the measured rms voltage [V], $\alpha$ is the system calibration coefficient $\left[\mathrm{A} \cdot \mathrm{m}^{2} \cdot \mathrm{V}^{-1} \cdot \mathrm{S}^{-1}\right], \mathrm{V}_{\mathrm{s}}$ is the sample volume $\left[\mathrm{m}^{3}\right], f$ is the frequency of ac fields $\left[\mathrm{s}^{-1}\right], \mathrm{H}_{r m s}$ is the rms magnetic field [A.m ${ }^{-1}$ ], and $\chi_{\text {ext }}$ is the volume susceptibility of the sample in SI units [dimensionless].

Rearranging equation (13) produces the formulation used to drive the external sample susceptibility, $\chi_{\text {ext }}$, from the experimental parameters:

$$
\chi_{e x t}=\frac{\alpha v_{r m s}}{V_{S} f H_{r m s}}
$$


The absolute accuracy of the measured susceptibility depends upon the accuracy with which each of the five parameters in eqn. (14) are determined.

The temperature dependence of the susceptibility of the samples is recorded upon warming up and zero field cooling, ZFC. Upon cooling through $\mathrm{T}_{\mathrm{c}}$, flux exclusion is often incomplete and pinned flux may remain in the sample that might contribute to the susceptometer signal. Anyhow, measurements upon warming or cooling, ac-susceptibility (with zero dc bias field) always measures shielding.

\section{Calibration Coefficient}

The calibration coefficient $\alpha$ relates the magnetic flux coupling between the sample and the sensing coil, so the exact value of $\alpha$ will be dependent on the sample size and geometry. The susceptometer pick-up coil can be calibrated analytically for spherical samples [16]. We use the dipole field of a uniformly magnetized sphere or a sample that is small with respect to the sensing coil. The magnetic moment $\mathrm{m}=\mathrm{M} \mathrm{V}$, where $\mathrm{M}$ is the magnetization and $\mathrm{V}_{\mathrm{s}}$ is the sample volume, and calculate the total flux $\phi$ through a thin pick-up coil of radius a, length $\mathrm{L}$, and $\mathrm{N}_{\mathrm{s}}$ turns. We assume $\mathrm{M}$ $=\chi_{e x t} H_{a}$ and $H_{a}=H_{o} \sin (2 \pi f t)$, where $\chi_{e x t}$ is the external susceptibility in SI units, $H_{o}$ and $f$ are the amplitude and frequency of the applied field $H$ a. Finally, using Faraday's law, $v=-\delta \Phi / \delta$ t, where, $v$, is the pick-up coil voltage, and get:

$$
\chi_{e x t}=v_{r m s}\left[(1 / 2 \mathrm{~L})^{2}+\mathrm{a}^{2}\right]^{1 / 2} /\left(\mathrm{N}_{\mathrm{s}} \mathrm{V}_{\mathrm{s}} \pi f \mu_{\mathrm{o}} H_{r m s}\right)
$$

comparing with equation (14), we can extract an expression for the calibration coefficient $\alpha\left[\mathrm{A} \cdot \mathrm{m}^{2} . \mathrm{V}^{-1} \cdot \mathrm{S}^{-1}\right]$ for a given pick-up coil:

$$
\alpha=\left[(1 / 2 \mathrm{~L})^{2}+\mathrm{a}^{2}\right]^{1 / 2} /\left(\mathrm{N}_{\mathrm{s}} \pi \mu_{\mathrm{o}}\right)
$$

Verification of the calculated coefficients has been done using $\mathrm{Ni}$ cylindrical material with Curie temperature $T_{c}=627 \mathrm{~K}$ [18]. The resultant experimental value has agreed to within the experimental uncertainties of a few percentages. This is consistent with the values obtained by other groups [19].

\section{Demagnetizing factor considerations}

Demagnetizing factors are important for the understanding of the susceptibility of superconductors and especially films. Experimental and theoretical work on demagnetizing factors for cylinders [20] and ellipsoids [21] 
can be found in the literature. For precision measurements or when the susceptibility is large, the measured susceptibility must be corrected for demagnetization effects. The internal magnetic field, $\mathrm{H}_{i n}$, which a magnetic material experience will not necessarily be identical to the external applied field, $\mathrm{H}_{e x t}$. The demagnetization factor relates the internal and external field in a uniformly magnetized material:

$$
\mathrm{H}_{i n}=\mathrm{H}_{\text {ext }}-\mathrm{N} \mathrm{M}
$$

where $\mathrm{N}$ is the demagnetizing factor $(0<\mathrm{N}<1)$ and $\mathrm{M}$ is the magnetization of the material. The demagnetization factor is a geometric effect and for thin films, $\mathrm{N}=0$ when the plane of the film is oriented parallel to the applied field and $\mathrm{N}=1$ when the film oriented perpendicular.

In an ac susceptometer, the measurement of susceptibility requires the application of $\mathrm{H}_{\text {ext }}$ and measurement of M. The susceptibility $\delta \mathrm{M} / \delta \mathrm{H}_{\text {ext }}$ is a characteristic of the sample and may be termed the external susceptibility $\chi_{\text {ext }}$. The internal susceptibility $\chi_{i n}$, characteristic of the material is $\delta \mathrm{M} / \delta \mathrm{H}_{\text {in }}$. The measured susceptibility should be converted to the true internal susceptibility. The two susceptibilities are related:

and

$$
\chi_{i n}=\mathrm{M} / \mathrm{H}_{i n}=\chi_{\text {ext }} /\left(1-\mathrm{N} \chi_{e x t}\right),
$$

$$
\chi_{\text {ext }}=\mathrm{M} / \mathrm{H}_{\text {ext }}=\chi_{\text {in }} /\left(1+\mathrm{N} \chi_{\text {in }}\right)
$$

When ac susceptibility is measured, $\chi_{\text {ext }}$ is a complex quantity, $\chi_{\text {ext }} \equiv$ $\chi_{\text {ext }}^{\prime}+\mathrm{i} \chi^{\prime \prime}{ }_{\text {ext }}$, and internal susceptibility is also complex: $\chi_{i n} \equiv \chi^{\prime}{ }_{i n}+\mathrm{i} \chi^{\prime \prime}{ }_{i n}$. When the two quantities $\chi_{\text {in }}$ and $\chi_{\text {ext }}$-the real parts and the imaginary parts- are separated, then ${ }^{(11,22)}$ :

$$
\begin{gathered}
\chi_{i n}^{\prime}=\frac{\left[\chi_{\text {ext }}^{\prime}-N\left(\chi_{\text {ext }}^{\prime 2}+\chi_{\text {ext }}^{\prime \prime 2}\right)\right]}{\left[N^{2}\left(\chi_{\text {ext }}^{2}+\chi_{\text {ext }}^{\prime 2}\right)-2 N \chi_{\text {ext }}^{\prime}+1\right]} \\
\chi_{\text {in }}^{\prime \prime}=\frac{\chi_{\text {ext }}^{\prime \prime}}{\left[N^{2}\left(\chi_{\text {ext }}^{\prime 2}+\chi_{\text {ext }}^{\prime \prime 2}\right)-2 N \chi_{\text {ext }}^{\prime}+1\right]}
\end{gathered}
$$

When $\mathrm{N} \approx 1$ and $\chi^{\prime}{ }_{e x t} \approx 1$ and $\chi^{\prime \prime}{ }_{\text {ext }} \approx 0$, as might occur for films in the normal state, $\chi^{\prime}{ }_{\text {in }}$ and $\chi^{\prime \prime}{ }_{\text {in }}$ diverge, causing severe scatter in $\chi^{\prime}{ }_{\text {in }}$ and $\chi^{\prime \prime}{ }_{i n}$. Such values of $\chi^{\prime}{ }_{e x t}$ and $\chi^{\prime \prime}{ }_{e x t}$ are not uncommon in actual measurements of thick films. 


\section{Susceptometer sensitivity}

Recalling eqn. (14) which describes the response of an acsusceptometer, one can find that the sensitivity of an ac-susceptometer depends upon $\alpha$ (a function of the pick-up coil geometry) and on the output precision of the ac-voltmeter, $v_{r m s}$, of the lock-in amplifier $\pm 1.0 \mathrm{~V}_{r m s}$. Usually, $v_{r m s}$ is proportional to $f$, so that, $\alpha v_{r m s} / f$, represent the magnetic moment precision in units of A.m ${ }^{2}$. Our present homemade susceptometer have $\alpha=2.8399\left[\mathrm{~A}^{-\mathrm{m}^{2}} . \mathrm{V}^{-}\right.$ $\left.{ }^{1} . \mathrm{s}^{-1}\right]$ and $v_{r m s} / f=2.5 \times 10^{-10} \mathrm{~V}$.s, giving a moment sensitivity of $7 \times 10^{-10} \mathrm{~A} . \mathrm{m}^{2}$. For comparison, a commercial vibrating sample magnetometer is designed to measure about $5 \times 10^{-8} \mathrm{~A} . \mathrm{m}^{2}$, the commercial SQUID magnetometers[11] can detect about $10^{-11} \mathrm{~A} \cdot \mathrm{m}^{2}$, while alternating-gradient-force magnetometers[23] can measure about $10^{-11}$ A.m ${ }^{2}$. The present sensitivity means that the precision in $\chi_{\text {ext }}$ is of the order $7 \times 10^{-10} /\left(V_{s} \mathrm{H}_{\mathrm{rms}}\right)$. This means that, the sample susceptibility can be measured more precisely if we have a larger sample or use a higher field.

\section{Results and discussion}

Following are our present experimental findings using the experimental facility given above. Two different ceramic samples of the compositions $\mathrm{YBa}_{2} \mathrm{Cu}_{3} \mathrm{O}_{7-\mathrm{x}}(\mathrm{YBCO})$ and $\mathrm{EuBa}_{2} \mathrm{Cu}_{3} \mathrm{O}_{7-\mathrm{x}}(\mathrm{EuBCO})$ were prepared by the usual procedure [24]. Fig. (2) shows the x-ray diffraction pattern of the final samples. It is clear that a single-phase composition is produced. A rectangular shaped sample for the magnetic susceptibility measurements was obtained by cutting and grinding the original pallet.

\section{Critical Temperature}

Usually the resistivity drop is characterizing the transition from a normal to the superconducting state. However, the magnetic manifestation of zero resistivity is that a material is a superconductor if it exhibits perfect diamagnetic shielding; that is, its susceptibility $\chi$ is exactly -1 (in SI units, where numerical results must be corrected for any sample demagnetization factor). Therefore an ac susceptibility measurement can be used to determine the critical temperature [25] $\mathrm{T}_{\mathrm{c}}$. Sometimes the onset temperature of transition, $\mathrm{T}_{\text {onset }}$, is used to characterize the transition temperature and thus the quality of the sample. Onset temperature is defined as the onset upon cooling, even if the measurement is made upon warming, which occur with the first sign of zeroresistance. However, $\mathrm{T}_{\text {onset }}$ is often connected to the Meissner shielding, resulting in decreasing of $\chi$, while $\chi$ " may remains zero in ideal superconductors with no apparent loss. 

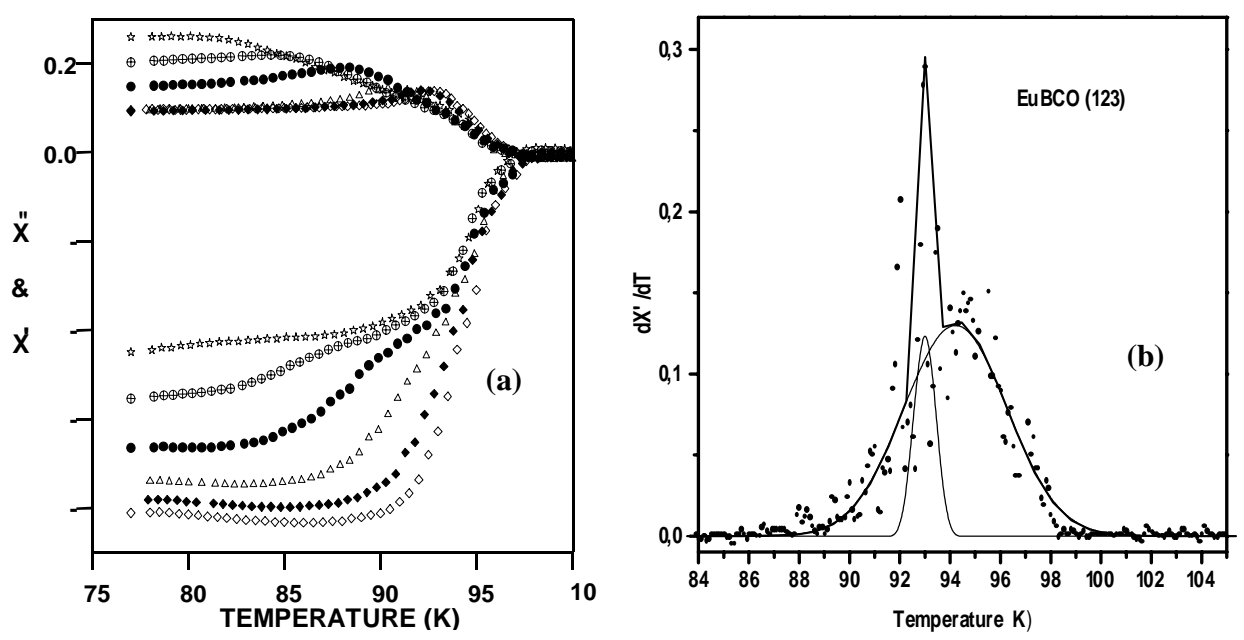

Fig. (4): Ac susceptibility ( $\chi$ ' and $\left.\chi{ }^{\prime \prime}\right)$ results for the superconducting $\mathrm{EuBa}_{2} \mathrm{Cu}_{3} \mathrm{O}_{7-\mathrm{x}}$ measured at different temperatures and applied magnetic field.

Fig. (3-a) shows the experimental result as obtained without any intended corrections. It can be shown that the onset temperature for YBCO sample is at $90.5 \mathrm{~K}$ as obtained by differentiating the $\chi$ ' against temperature curve. This result as shown in Fig. (3-b) proves the precision of our temperature measurements setting. On the other hand EuBCO susceptibility measurements are shown in Fig. (4-a), at different applied ac field at $1.014 \mathrm{kHz}$.

\section{Penetration depth $\delta$ and resistivity $\rho_{n}$}

Linear diffusion model is treated in textbooks of classical electrodynamics (for example, see ref. 26). Because of the linear character of the diffusion, higher harmonics are not generated in the pick-up signal. For a slab of width $2 \mathrm{R}$ in a parallel magnetic field Kes et. al. [27] drived an expression for the complex susceptibility in terms of the width and the penetration (skin) depth $\delta$ :

$$
\begin{aligned}
& \chi^{\prime}=\left[\frac{\boldsymbol{\delta}}{2 R}\right]\left[\frac{\sinh (2 R / \boldsymbol{\delta})-\sin (2 R / \boldsymbol{\delta})}{\cosh (2 R / \boldsymbol{\delta})+\cos (2 R / \boldsymbol{\delta})}\right]-1 \\
& \chi^{\prime \prime}=\left[\frac{\boldsymbol{\delta}}{2 R}\right]\left[\frac{\sinh (2 R / \boldsymbol{\delta})+\sin (2 R / \boldsymbol{\delta})}{\cosh (2 R / \boldsymbol{\delta})+\cos (2 R / \boldsymbol{\delta})}\right]
\end{aligned}
$$

The expressions for a spherical sample are of similar form [3]. The corresponding formulas for a cylinder can be found in the literature [28]. The 
experimental results are shown in Fig.(5). The linear regime is dissipative, which means that a loss of magnetic energy appears and the peak in $\chi^{\prime \prime}$ will be reached when $\delta=0.556 \mathrm{R}$ in the case of a slab of width $2 \mathrm{R}$ (equal to $0.8 \mathrm{~mm}$ ).
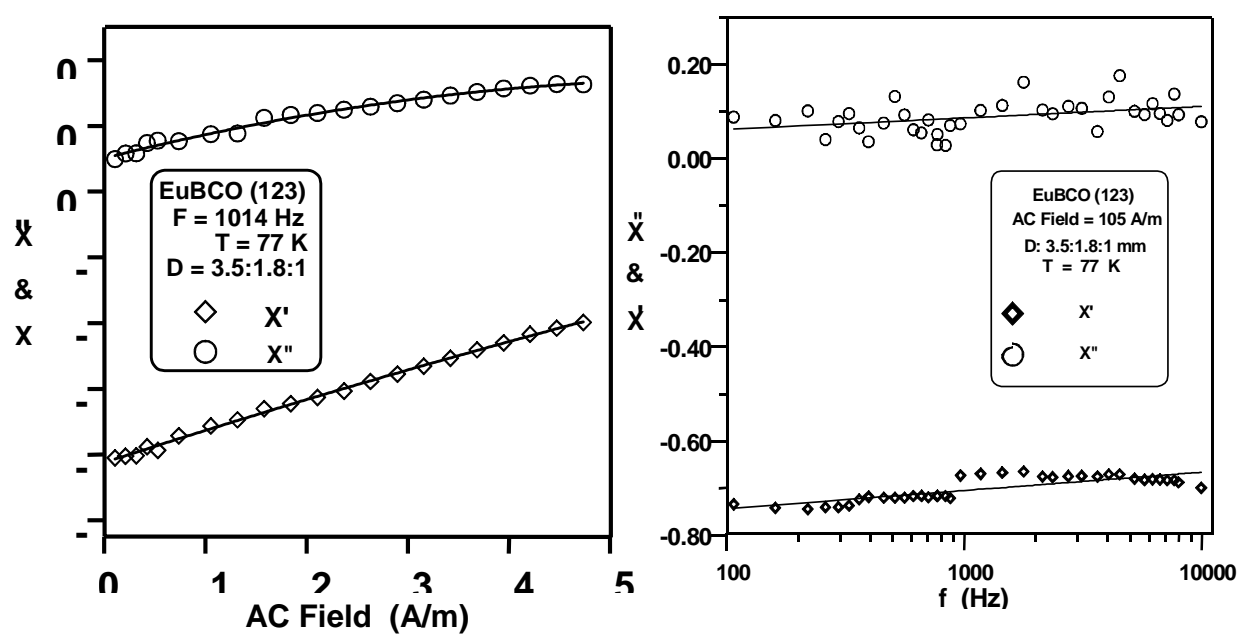

Fig. (5): (a) Variations of $\chi$ ' and $\chi$ " as a function of applied ac magnetic field at liquid nitrogen temperature $(77 \mathrm{~K})$ and constant frequency of $1.014 \mathrm{kHz}$.

(b) Variation of $\chi^{\prime}$ and $\chi$ ' as a function of applied frequency at constant applied external ac field of $105 \mathrm{~A} / \mathrm{m}$ at $77 \mathrm{~K}$.

In the case of linear diffusion the resistivity $\rho_{\text {lin }}$ is the only material parameter controlling the flux dynamics. The resistivity $\rho_{\text {lin }}(\mathrm{B})$ defines the relation between the electric field and the current density [2] is:

$$
\mathrm{J}(\mathrm{B}, \mathrm{E})=\mathrm{E} / \rho_{\text {lin }}(\mathrm{B})
$$

Where

$$
\rho_{l i n}(\mathrm{~B})=\mathrm{E}_{\mathrm{c}}(\mathrm{B}) / \mathrm{J}_{\mathrm{c}}(\mathrm{B})
$$

The ac field amplitude $\mathrm{B}_{\mathrm{o}}(\mathrm{x})$ is decaying roughly exponentially [29]:

$$
\mathrm{B}_{\mathrm{o}}(\mathrm{x})=\mathrm{B}_{\mathrm{a}} \exp (-\mathrm{x} / \delta)
$$

The characteristic space scale of the ac field decay is: 


$$
\delta=\left[\frac{2 \rho_{l i n}}{\mu \omega}\right]^{1 / 2}
$$

Important mechanisms of the flux dynamics belonging to this case are normal-state eddy currents, linear flux flow and thermally assisted flux flow. They differ in the value of the linear resistivity [29]. For eddy currents the apparent susceptibility is due to that currents, not to the magnetic properties of the material, much as the magnetic susceptibility of a superconductor arises from shielding currents. Thus, the resistivity in eqn. (24) is now equal to the normal resistivity $\rho_{\text {lin }}=\rho_{n}$. Therefore the penetration depth $\delta$ enter the expression (20) can be expressed as:

$$
\delta=\left[\frac{\rho_{n}}{\pi f \mu}\right]^{1 / 2}
$$

where $f$ is the applied frequency and $\mu=\mu_{\mathrm{o}}$ for a non-magnetic material. Knowing $\delta(\mathrm{T})$ from eqn. (20) at different temperatures as shown in Fig. (6) together with the theoretical fitting applying the well-known relation [2];

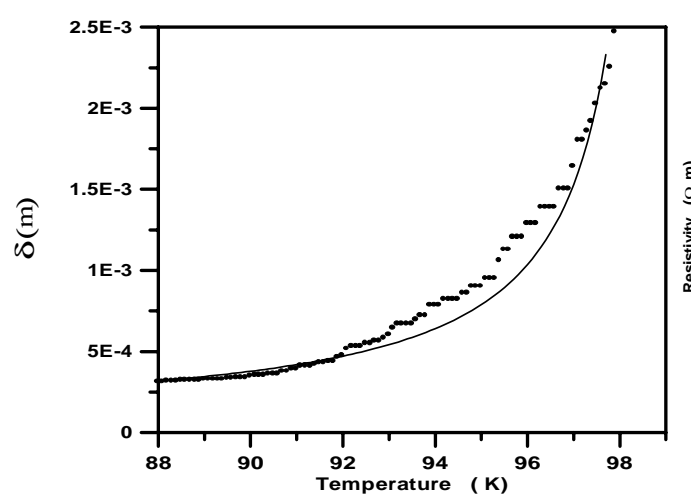

Fig. (6)

Penetration depth $(\bullet)$ near the critical temperature applying eqn. (20a) The continuos curve is due to the application of eqn (25b).

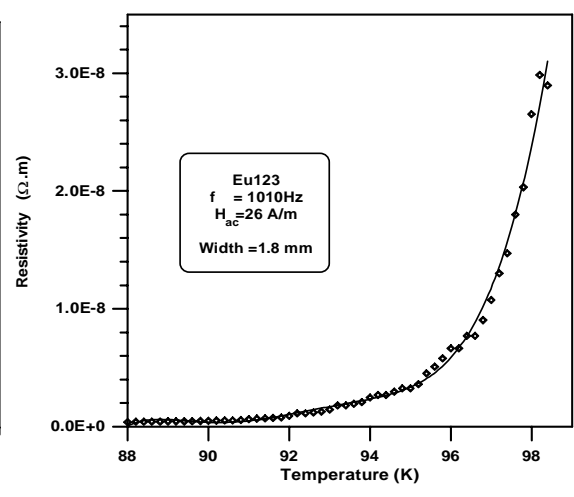

Fig. (7)

Calculated resistivity near to the critical temperature according to eqn. (25a). The solid curve is guide to the eye.

$$
\delta=\delta_{o} *\left[1-\left(\frac{T}{T_{c}}\right)^{4}\right]^{-1 / 2},
$$


one can estimate numerically the normal resistivity [30] $\rho_{n}$ as the temperature approaches $\mathrm{T}_{\mathrm{c}}$. Fig. (7) shows the results obtained for the EuBCO sample in the vicinity of the critical temperature, as extracted from the experimental penetration depth $\delta$ applying eqns. (20a and 25) respectively.

\section{Critical current density $\mathbf{J}_{\mathbf{c}}$}

The behavior of a superconducting material able to pin the flux line lattice is well described by the Bean critical-state model [31]. The flux density gradient in the critical state is taking the form:

$$
\frac{\partial B}{\partial x}=\mp \mu_{\circ} J_{c}(B)
$$

Therefore, the material is characterized by the critical current density $\mathrm{J}_{\mathrm{c}}$.

Suppose that $J_{c}$ is constant independent on the $A C$ magnetic field. This approximation is valid if $B_{d}>B_{a}$ because $J_{c}(B) \approx J_{c}\left(B_{d}+B_{a}\right)$. At the moment when the ac magnetic field (eqn.5) reaches a maximum value (i.e. at $\omega \tau=0,2 \pi$, $4 \pi, \ldots, 2 n \pi)$, the ac flux density profile takes the form:

$$
B(x, 0)=B_{a}-\mu_{o} J_{c} x
$$

Field penetration is stopped at depth:

$$
x_{\mathrm{c}}=\frac{B_{a}}{\mu_{o} J_{c}}
$$

Let us consider the simplest case when the flux pinning is the only mechanism controlling the flux dynamics, and the current- carrying capability is supposed to remain constant throughout the sample. In the case of a slab with thickness $2 \mathrm{R}$, the simplest estimation of $\mathrm{J}_{\mathrm{c}}$ can be performed by knowing that the maximum of $\chi^{\prime \prime}$ is reached at a particular value of the AC field penetration depth [32]: $x_{\mathrm{c}}=\sqrt{2} R$, so that;

$$
\begin{aligned}
& B_{a}=\sqrt{2} \mu_{o} J_{c} R \\
& \left(\text { At maximum } \chi^{\prime \prime} \text { where } \mathrm{H}_{\mathrm{m}}=\mathrm{H}_{\mathrm{p}}\right. \text { ) }
\end{aligned}
$$

Using eqn.29, the $\mathrm{J}_{\mathrm{c}}(\mathrm{T})$ dependence can be found by measuring $\chi^{\prime \prime}$ at different amplitudes, $B_{a}$, of the ac field that leads to finding the peaks at different temperatures [23]. It is worth mentioning that in this approach we use only one point from the whole susceptibility curve, see Fig. (8). 
To process a large portion of the susceptibility data it is more effective to use the following formula for the real and imaginary parts of the external susceptibility $[2,11,34]$. For a slab (width $2 \mathrm{R}$ ) in a parallel magnetic field;
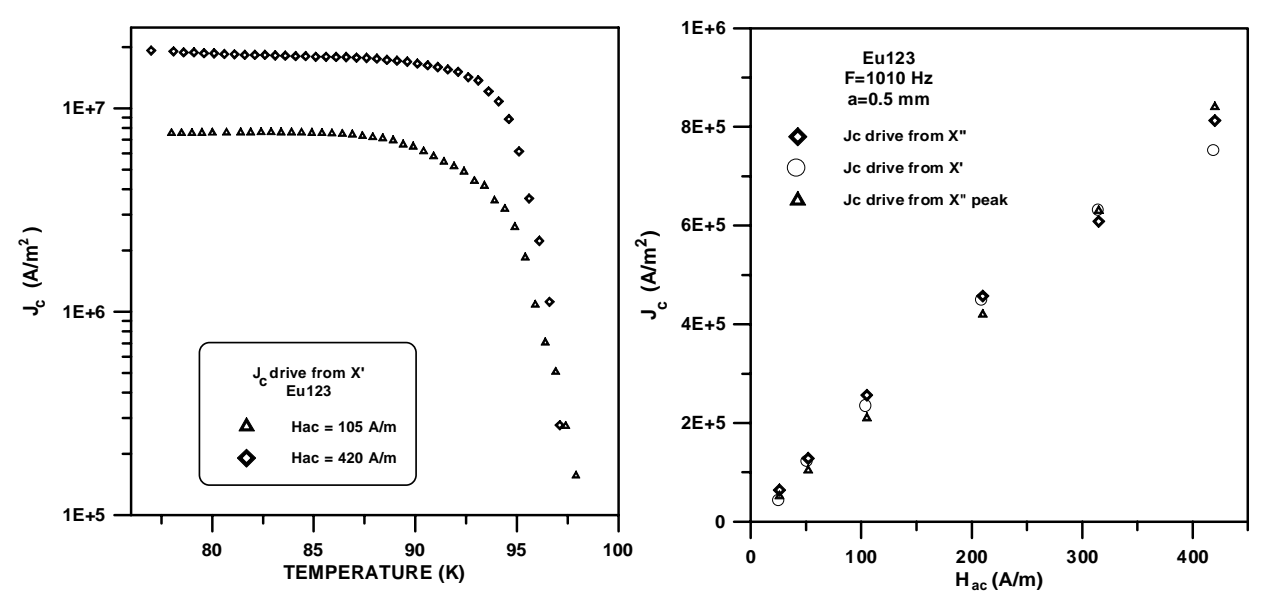

Fig. (8):(a) Critical current density at different temperatures and two selected external rms applied magnetic fields, according to eqn. (30a).

(b) Critical current density as a function of applied magnetic field as obtained applying eqns. $(29,30 \mathrm{a}$ and $30 \mathrm{~b})$ for comparison.

$$
\begin{aligned}
& \chi^{\prime}=\left\{\begin{array}{lll}
\frac{y}{2}-1 & \text { for } & 0 \leq \mathrm{y} \leq 1.0 \\
\frac{1}{\pi}\left[\left(\frac{y}{2}-1\right) \cos ^{-1}(1-2 y)+\left(-1+\frac{4}{3 y}-\frac{4}{3 y^{2}}\right)(y-1)^{1 / 2}\right] & \text { for } & 1.0 \leq \mathrm{y}
\end{array}\right. \\
& \chi^{\prime \prime}=\left\{\begin{array}{lll}
\frac{2 y}{3 \pi} & \text { for } & 0 \leq \mathrm{y} \leq 1.0 \\
\frac{1}{3 \pi}\left(\frac{6}{y}-\frac{4}{y^{2}}\right) & \text { for } & 1.0 \leq \mathrm{y}
\end{array}\right.
\end{aligned}
$$

Where the auxiliary variable $y=\frac{x_{c}}{R}=\frac{B_{a}}{\mu_{o} J_{c} R}$.

\section{Conclusions}

We have described our design, calibration, sensitivity determination, and operation of the laboratory built low-frequency ac-susceptibility 
measurement using local facilities. The designed ac-susceptometer is based on the mutual inductance method, and consists of two identical primary excitation field coils and two identical secondary pick-up coils. One of the most useful features of our laboratory-built ac susceptometer is that the real, or in-phase, component $\left(\chi^{\prime}\right)$, and the imaginary, or out of-phase, component $\left(\chi^{\prime \prime}\right)$, of the complex susceptibility can be measured independently applying a computer program designed specially for this purpose.

Our presented homemade susceptometer have $\quad \alpha=2.8399\left[\mathrm{~A} \cdot \mathrm{m}^{2} . \mathrm{V}^{-1}\right.$. $\mathrm{s}^{-1}$ ] and $v_{r m s} / f=2.5 \times 10^{-10}$ V.s, giving a moment sensitivity of $7 \times 10^{-10}$ A.m. This moment sensitivity is comparable with other magnetic techniques. The present sensitivity means that the precision in $\chi_{e x t}$ is of the order $7 \times 10^{-10} /\left(V_{s}\right.$ $\left.\mathrm{H}_{\mathrm{rms}}\right)$. This means that, the sample susceptibility can be measured more precisely if we have a larger sample or use a higher field.

The formulas for susceptibilities corresponding to the models of linear diffusion and critical state have been used in the recognition of the mechanism controlling the flux dynamics and in the determination of the superconducting properties for $\mathrm{YBa}_{2} \mathrm{Cu}_{3} \mathrm{O}_{7-\mathrm{x}}(\mathrm{YBCO})$ and $\mathrm{EuBa}_{2} \mathrm{Cu}_{3} \mathrm{O}_{7-\mathrm{x}}(\mathrm{EuBCO})$. We have seen that, important parameters such as the critical temperature, normal resistivity, critical current density, and penetration depth can be deduced from the magnetic susceptibility measurements of the superconductor.

\section{References}

1. Lee L.P, Char k, Colclough M.S and Zaharchuk G, Appl. Phys. Lett. 59, 3051 (1991).

2. Gomory F, Supercond. Sci. Technol 10, 523 (1997).

3. Couach M. and Khoder A.F, in "Magnetic Susceptibility of Superconductors and other Spin Systems" ed. R.A. Hein, T.L. Francavilla and D.H. Liebenberg, Plenum, New York (1992) P.35.

4. John Crangle, "Solid State Magnetism", Edward Arnold, Great Britain (1991), PP: 57-65.

5. Hein R.A and Falge R.L. Jr, Phys. Rev. 123, 407 (1961).

6. Griffiths D.J, Koch C.C. and Charlesworth J.P, Phil. Mag. 33, 505 (1976).

7. Maxwell E. and Strongin M, Phys. Rev. Lett. 10, 212 (1963).

8. Ishida T. and Mazaki H, Phys. Rev. B 20, 131 (1979).

9. Campbell A.M, IEEE Trans. Appl. Supercond. 5, 682 (1995).

10. Stoppard O. and Gugan D, Physica C 241, 375 (1995). 
11. Goldfarb R.B, Lelental $M$, and Thompson C.A, in "Magnetic Susceptibility of Superconductors and other Spin Systems" ed. R.A. Hein, T.L. Francavilla and D.H. Liebenberg, Plenum, New York (1992) P.49.

12. Filippi j., Pugnat $P$, Barbara $B$, Ingold $M$, Bourgault $D$ and Tournier R, Physica C 235-240, 3138 (1994).

13. Clem J.R, Physica C 153-155, 50 (1988).

14. Jiang H and Bean C.P, Appl. Supercond. 2, 689 (1994).

15. Gomory F, Thermochim. Acta 174, 299 (1991).

16. Whitmore S.C, Ryan S.R, and Sanders T.M, Rev. Sci. Instrum. 49, 1579 (1978).

17. Edgar A, and Quilty J.W, Am. J. Physics 61, 943 (1993).

18. Leger J.M, Susse C, Epain R, and Vodar B, Solid State Commun. 4, 197 (1966).

19. Couach M, Khoder A.F, and Monnier F, Cryogenics 25, 695 (1985).

20. Crabtree G.W, Phys. Rev. B 16, 1117 (1977).

21. Osborn J.A, Phys. Rev. 67, 351 (1945).

22. Ferreirinho J, Lee S.J, Campbell S.J, and Calka A, J. Magn. Magn. Mater. 88, 281 (1990).

23. Flanders P.J, J. Appl. Phys. 63, 3940 (1988).

24. Li S, Hayri E.A, Ramanujachary K.V, and Martha Greenblatt, Phys. Rev. B 38,2450 (1988).

25. Opagiste C, Couach M, Khoder A, Triscone G, Jorda J.L and Cohen-Adad M.T, Physica B 194-196, 1809 (1994).

26. Smythe W.R, In "Static and Dynamic Electricity", New York, McGraw-Hill (1950) P. 390.

27. Kes P.H, Aarts J, van den Berg J, van der Beek C.J and Mydosh J.A, Supercond. Sci. Technol. 1, 242 (1989).

28. Clem J.R, Kerchner H.R and Sekula T.S, Phys. Rev. B 14, 1893 (1976).

29. Brandt E.H, Supercond. Sci. Technol. 5, 525 (1992).

30. Polichetti M, Pace S, Reggiani L and Testa A.M, $4^{\text {th }}$ Euro Ceramics vol. 7 , ed. a. Barone, D. Fiorani and A. Tampieri (Rome: Faenza), (1996) P. 85.

31. Bean C.P, Rev. Mod. Phys. 36, 31 (1964).

32. Gomory F, Solid State Commun. 70, 879 (1989).

33. Widder W, Bauernfeind L, Stebani M and Braun H.F, Physica C 249, 78 (1995).

34. Ji L, Sohn H.R, Spalding G.C, Lobb C.J. and Tinkham M, Phys. Rev. B 40, 10936 (1989). 